\title{
The IceCube Neutrino Telescope
}

\author{
Thomas K. Gaisser ${ }^{1}$ for the IceCube Collaboration ${ }^{2}$ \\ ${ }^{1}$ Bartol Research Institute and Dept. of Physics and Astronomy \\ University of Delaware, Newark, DE 19716 USA \\ email: gaisser@bartol.udel.edu \\ ${ }^{2}$ See http://icecube.wisc.edu
}

\begin{abstract}
Construction of IceCube at the Amundsen-Scott South Pole Station was completed at the end of 2010 after eight construction seasons. The detector consists of 5,160 digital optical modules on 86 cables with 60 modules each, viewing in total a cubic kilometer of ice between 1,450 and 2,450 meters below the surface. IceCube includes a sub-array called DeepCore consisting of 8 special cables, and providing a more densely instrumented region with a lower energy threshold in the deep center of the array. IceCube also includes an air shower array called IceTop directly above the deep detector. Optical modules in all three components of the detector are fully integrated into a single data acquisition system. Data taking and analysis began during construction and continues with the completed detector. This paper describes recent results from IceCube.
\end{abstract}

Keywords. Neutrinos, cosmic rays

\section{Introduction}

The idea of detecting neutrinos in a large instrumented volume of water dates back to work of Markov (1960), Greisen (1960) and Reines (1960). The idea is to use the Cherenkov light generated by charged particles produced when neutrinos interact in a large, transparent volume of water (or ice). Development of the original idea followed two paths. One was densely instrumented detectors aimed at the $\mathrm{GeV}$ region motivated originally by the search for proton decay. This effort paid off in a big way with the observation of neutrinos from SN1987A by Kamioka (Hirata et al., 1987) and IMB (Bionta et al., 1987) and the discovery of neutrino oscillations by Super-Kamiokande (Fukuda et al., 1998) and SNO (Jelley, MacDonald \& Robertson, 2009).

The other path is motivated by the quest to detect high-energy neutrinos of astrophysical origin above the steeply falling spectrum of neutrinos produced locally by interactions of cosmic rays in the atmosphere. For this objective, the instrumented volume should be as large as possible and the detectors placed as far apart as allowed by transparency of the medium. IceCube is in this line, started in the 1970's by the DUMAND Project, an effort to deploy an array of photomultipliers in the ocean near Hawaii. Although DUMAND itself was realized only with the deployment of a single string from a ship for several days in 1987 (Babson et al.,1990), the DUMAND effort in the seventies and eighties set the stage for high energy neutrino astronomy. The Lake Baikal detector in Siberia (Balkanov et al., 1999) and the ANTARES detector in the Mediterranean (Ageron, et al., 2011) are the two large neutrino telescopes currently operating in water.

One of the first papers to discuss the possibility of using ice rather than water as the detector medium (Halzen et al., 1989) was presented in 1989 at a conference on prospects for astrophysics in Antarctica (AIP, 1989). The meeting was hosted by Martin Pomerantz and the Bartol Research Institute at Delaware with support from the NSF Office of Polar Programs. Plans for AMANDA (Antarctica Muon and Neutrino Detector 


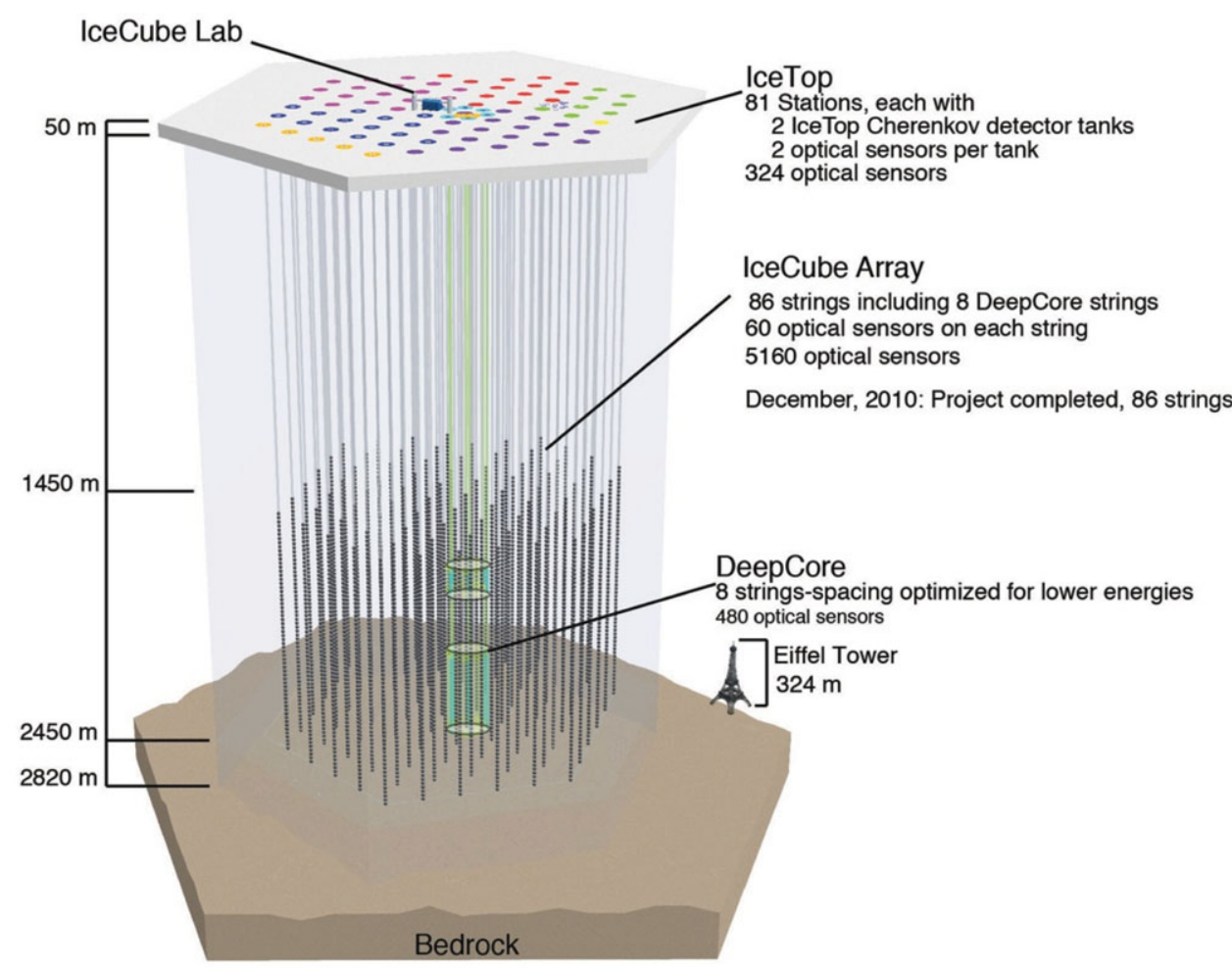

Figure 1. The IceCube Neutrino Telescope.

Array) developed in the decade following this meeting. It is interesting to note that the Center for Astrophysical Research in Antarctica (CARA) for millimeter and submillimeter astronomy at the South Pole traces its origin to the same meeting. Five years later the Martin A. Pomerantz Observatory (MAPO) was inaugurated at the South Pole to house the astronomy experiments and AMANDA. The South Pole Telescope and IceCube are both descendants of that era. Further description of AMANDA (IceCube, 2009a) as the predecessor of IceCube are presented in a previous paper in these Proceedings (Karle, 2012).

Construction of IceCube was achieved in eight Antarctic seasons, starting in 2003/04 with the first shipments of equipment (Karle, 2012). The first deep hole was drilled and the first IceCube cable deployed in January, 2005 along with the first 4 stations of IceTop. The completed IceCube has been in operation since May 2011 with 86 strings between 1.45 and $2.45 \mathrm{~km}$ below the surface. Each string carries 60 digital optical modules (DOMs). On the surface, near the top of each string is a pair of tanks filled with clear ice and instrumented with two DOMs each. These 162 tanks at 81 stations constitute a square kilometer air shower array that is fully integrated into the IceCube data acquisition system. IceCube is thus a three-dimensional cosmic-ray detector as well as a neutrino telescope, as illustrated in Fig. 1.

\section{Motivation: neutrinos as a probe of cosmic-ray origin}

The principal purpose of IceCube is to find high-energy neutrinos of astrophysical origin to probe the origin of cosmic rays. In the Milky Way, this means searching for neutrinos associated with supernova remnants, active star forming regions or other possible sources 

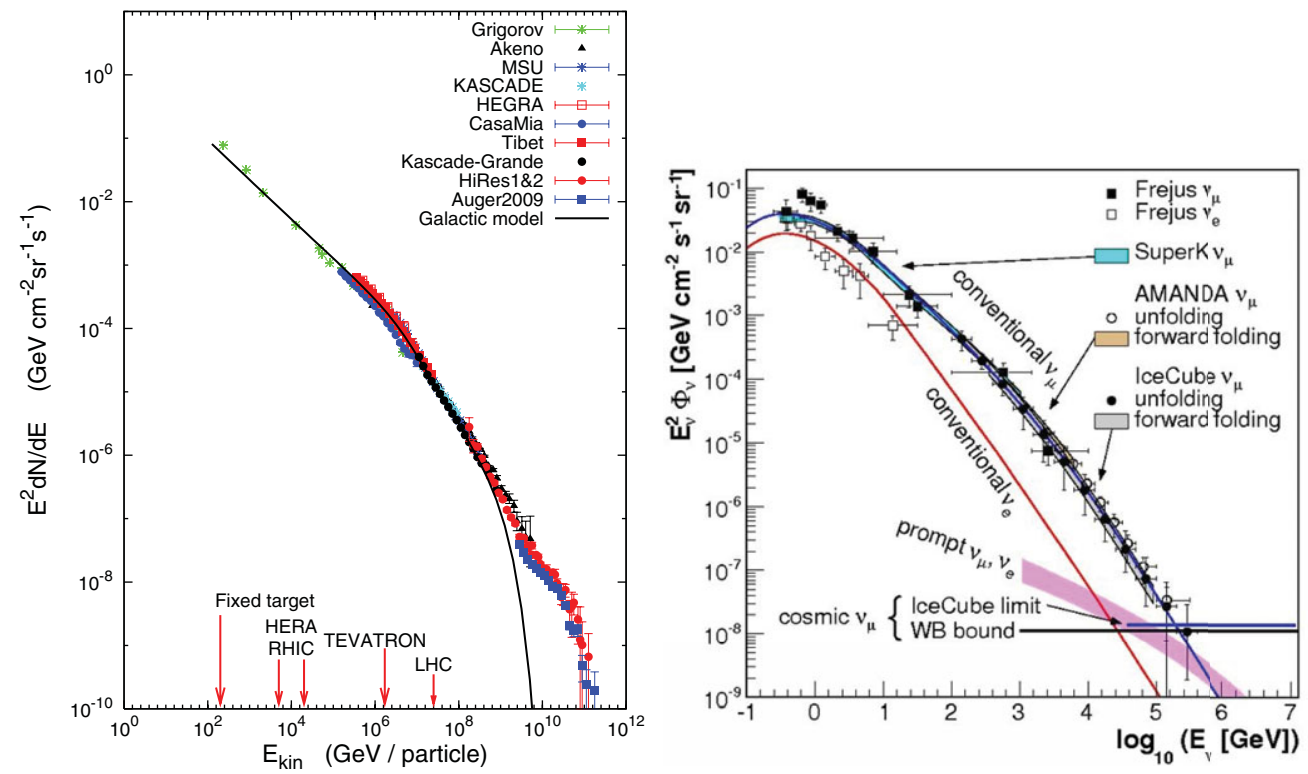

Figure 2. Left: The high-energy cosmic-ray spectrum. Solid line shows a possible model for fraction of observed cosmic rays from sources in the Milky Way. The plot is adapted from Gaisser (2012) where the references for the data are given. Right: Atmospheric $\nu_{\mu}$ spectrum and astrophysical limits.

of galactic cosmic rays. In the case of extra-galactic cosmic rays, likely sources are gammaray bursts (GRB) and active galaxies. Neutrinos and gamma-rays, being electrically neutral, would point back to the sources of the processes that produce them. Protons and nuclei generate both photons and neutrinos if they interact in the sources or with the surrounding medium to produce pions. Neutral pions decay to photons, while charged pions produce neutrinos. Neutrinos can only be of hadronic origin, so they directly reflect cosmic-ray acceleration. The relation of photons to cosmic rays is more complex. Since electrons are more efficient radiators than protons, they are often the direct progenitors of gamma-rays. In addition, photons are likely to cascade on their way out of the sources and during propagation. To summarize, photons are abundant but complicated to interpret, while neutrinos are clean but rare.

The simplicity of the connection between neutrinos and cosmic-rays makes it possible to estimate from the cosmic-ray spectrum the level at which high-energy astrophysical neutrinos from extra-galactic sources may be expected (Halzen, 2012). Figure 2 (left) shows a summary of measurements of the cosmic ray spectrum. The solid line shows a model of the galactic cosmic rays (Gaisser, 2012). The model is not unique, but it illustrates one class of models by Berezinsky et al. (2006) in which the galactic contribution of cosmic rays ends before the ankle in the spectrum around $3 \times 10^{18} \mathrm{eV}$. Another possibility (Allard et al., 2011) is that the galactic contribution extends somewhat higher in energy so that the ankle marks the transition to the extragalactic contribution.

In either case, by $10^{19} \mathrm{eV}$, the observed spectrum is expected to be dominated by sources outside the Milky Way. This assumption is also consistent with the nearly isotropic distribution of cosmic rays of this energy and their lack of correlation with the structure of our galaxy. This energy is also below the energy of $5 \times 10^{19} \mathrm{eV}$ above which energy losses by pion photoproduction of protons and photodisintegration of nuclei become important. We can therefore use the observed energy flux in this energy range 
to estimate the energy content of the extragalactic cosmic rays and hence the power required of their sources. Reading off the plot, the energy flux at $10^{19} \mathrm{eV}$ is

$$
E \frac{\mathrm{d} N}{\mathrm{~d} \ln E} \approx 2 \times 10^{-8} \mathrm{GeV} \mathrm{cm}^{-2} \mathrm{sr}^{-1} \mathrm{~s}^{-1}
$$

which corresponds to an energy density of $\frac{4 \pi}{c} E \frac{\mathrm{d} N}{\mathrm{~d} \ln E} \approx 1.3 \times 10^{-20} \mathrm{erg} \mathrm{cm}^{-3}$, and to a differential power required of the sources,

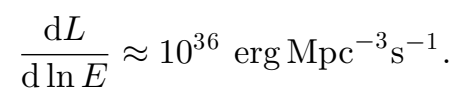

The simple estimate of Eq. 2.2 is obtained by dividing the observed energy density by the Hubble time, neglecting possible effects of source evolution. The total energy required is $\sim 10^{37}$ erg $\mathrm{Mpc}^{-3} \mathrm{~s}^{-1}$ or greater because the source spectrum must extend over several orders of magnitude. This leads to the estimated power per individual source (Table 1).

\begin{tabular}{l|l|l|}
\hline & Source density or rate & av. power required per src. \\
\hline Galaxies & $3 \times 10^{-3} \mathrm{Mpc}^{-3}$ & $5 \times 10^{39} \mathrm{erg} / \mathrm{s}$ \\
Galaxy clusters & $3 \times 10^{-6} \mathrm{Mpc}^{-3}$ & $5 \times 10^{42} \mathrm{erg} / \mathrm{s}$ \\
AGN & $1 \times 10^{-7} \mathrm{Mpc}^{-3}$ & $1 \times 10^{44} \mathrm{erg} / \mathrm{s}$ \\
GRB & $300 \mathrm{GRB} /\left(\mathrm{Gpc}^{3} \mathrm{yr}\right)$ & $10^{51} \mathrm{erg} / \mathrm{burst}$ \\
\hline
\end{tabular}

Table 1. Power requirements for some potential sources of ultra-high energy cosmic rays.

In addition to the energy requirement, potential sources also need to satisfy the Hillas (1984) relation, which requires the product of magnetic field and size of the source to be large enough to accelerate particles to $>10^{20} \mathrm{eV}$. Active galaxies and GRBs are considered the most likely candidate sources. It is important to note that active galaxies appear on the Hillas plot in two ways. Both active galactic nuclei (AGN) and giant radio galaxies are potential sources. The acceleration could occur inside the jets of AGN or far out in the intergalactic medium at the termination of the jets. The expectation for neutrinos would be quite different in the two cases, with little production if the acceleration occurs in the diffuse intergalactic medium.

If the acceleration occurs inside the jets of AGN or GRB, then one possible scenario is that the protons being accelerated are contained in the acceleration region by the magnetic fields required for the acceleration mechanism to work. Protons would interact with the intense radiation fields by photoproduction, with the main channels being

$$
\begin{aligned}
& p+\gamma \rightarrow \Delta^{+} \rightarrow p+\pi^{0} \rightarrow p+\gamma \gamma \text { and } \\
& p+\gamma \rightarrow \Delta^{+} \rightarrow n+\pi^{+} \rightarrow n+\mu^{+}+\nu_{\mu}
\end{aligned}
$$

followed by muon decay to $e^{+} \bar{\nu}_{\mu} \nu_{e}$. On average, each neutrino would carry $7 \%$ of the energy of the neutron (Ahlers et al. 2005). The neutrons may escape and decay to produce the extragalactic cosmic rays. Attributing the full observed flux of Eq. 2.1 to the escaping neutrons then predicts

$$
E_{\nu} \frac{\mathrm{d} N_{\nu}}{\mathrm{d} \ln E_{\nu}} \approx 1.4 \times 10^{-9} \mathrm{GeV} \mathrm{cm}^{-2} \mathrm{sr}^{-1} \mathrm{~s}^{-1}
$$

per flavor assuming oscillations equalizes the three different neutrino flavors. 


\section{Neutrinos in IceCube}

IceCube classifies neutrino events in two categories, track-like and cascades. Tracklike events are produced by charged current interactions of muon neutrinos. The event rate in this channel is a convolution of the neutrino flux with the neutrino cross section, the detector response and the range of the muon. At high energy the Earth becomes opaque to neutrinos, first for vertically upward neutrinos $(\sim 30 \mathrm{TeV})$ and then for more horizontal events $(\sim \mathrm{PeV})$. The rate of neutrino induced events is a convolution of the flux with the effective area of the detector. For $\nu_{\mu}$ in the charged current channel

$$
A_{\text {eff }}\left(\theta, E_{\nu}\right)=\epsilon(\theta) A(\theta) P_{\nu}\left(E_{\nu}, E_{\mu}\right) \exp \left\{-\sigma_{\nu}\left(E_{\nu}\right) N_{A} X(\theta)\right\},
$$

where $X(\theta)$ is the slant depth $\left(\mathrm{g} / \mathrm{cm}^{2}\right)$ along a zenith angle $\theta>90^{\circ}, N_{A}$ is Avogadro's number, $\sigma_{\nu}$ is the neutrino cross section and $\epsilon(\theta)$ a reconstruction efficiency.

$$
P_{\nu}\left(E_{\nu}, E_{\mu}\right)=N_{A} \int_{E_{\mu}}^{E_{\nu}} \mathrm{d} E_{\mu}^{*} \frac{\mathrm{d} \sigma_{\nu}\left(E_{\nu}\right)}{\mathrm{d} E_{\mu}^{*}} R\left(E_{\mu}^{*}, E_{\mu}\right)
$$

is the probability that a muon produced with energy $E_{\mu}^{*}$ reaches the detector with energy $E_{\mu}$ sufficient to trigger the detector. The typical ranges are $\sim 5$ and $\sim 15 \mathrm{~km}$ for muons with $10 \mathrm{TeV}$ and $1 \mathrm{PeV}$ respectively. Because of the large muon range, the efficiency for detecting $\nu_{\mu}$ is significantly greater than for the cascade channel. The energy deposited in the detector by a throughgoing muon is only a fraction of the energy of the parent $\nu_{\mu}$, so the visible energy is only statistically related to the neutrino energy. Above a $\mathrm{TeV}$, stochastic energy losses become dominant for muons, so the deposited energy is proportional on average to the energy of the muon at the detector.

Cascade events can be neutral current interactions of any flavor or charged current interactions of $\nu_{e}$ or $\nu_{\tau}$. In this case, the muon range in Eq. 3.1 is replaced by a fraction of the linear dimension of the instrumented volume, sufficiently $<1 \mathrm{~km}$ to satisfy a containment criterion. Thus, for equal fluxes, rates of cascade events will be correspondingly lower than track-like events. If the cascade is a charged current interaction of a $\nu_{e}$ or $\nu_{\tau}$, however, the visible energy in the cascade gives a direct measurement of the neutrino energy. The $\langle\Gamma c \tau\rangle$ for a $\tau$ lepton at $1 \mathrm{PeV}$ is $50 \mathrm{~m}$, compared the string spacing IceCube of 125 meters. Therefore it should be possible to discern the characteristic double bang structure (Learned \& Pakvasa, 1995) as an elongation of the cascade somewhere above this energy.

Most of the events in IceCube are $\sim \mathrm{TeV}$ muons produced by cosmic-ray interactions in the atmosphere above the detector. To find neutrinos, the Earth is used as a filter by selecting upward-moving tracks. The rate of events from atmospheric neutrinos producing muons from below the horizon is at the level of one part per million compared to the downward background. There is therefore a large contamination of mis-reconstructed muon events in the initial online selection. After applying cuts on track quality to reject accidentally coincident downward muons and other badly reconstructed events, a clean sample of neutrinos is found. These are mostly atmospheric, neutrino-induced muons.

\subsection{Atmospheric neutrinos}

Figure 2 (right) shows the fluxes of atmospheric $\nu_{\mu}+\bar{\nu}_{\mu}$ obtained from two analyses of data from IceCube when it was half finished (IC-40). One uses an unfolding method to relate the observed distribution of visible energy to the muon and hence to the neutrino that produced it (IceCube, 2011a). The other ((IceCube, 2011b) uses a forward folding method with the same physics but fitting parameters that describe the neutrino spectrum to the observed distribution of energy deposited in the detector. The parameters are the 

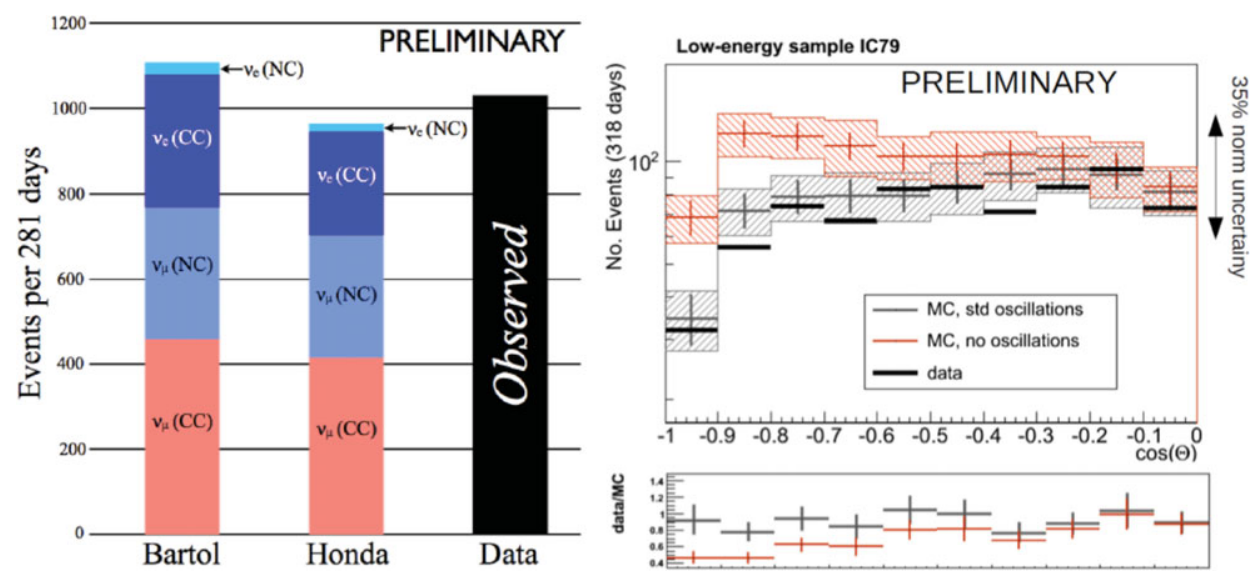

Figure 3. Neutrinos in DeepCore. Left: expectation for cascade events classified by channel compared to observation. Right: Effect of oscillations in the atmosphere on low-energy track-like events.

normalization and slope of the atmospheric neutrino spectrum, the normalization of the prompt neutrinos from decay of charm and the normalization of an astrophysical component assumed to have an $E^{-2}$ spectrum. The solid lines show upper limits on astrophysical neutrinos that will be discussed below. Also shown in the figure are results from IceCube's predecessor, AMANDA (IceCube, 2009b) and (IceCube, 2010b) along with earlier results from Frejus and SuperKamiokande.

In the $\mathrm{GeV}$ region there are approximately twice as many muon neutrinos produced in the atmosphere as electron neutrinos as a consequence of the $\pi \rightarrow \nu_{\mu}+\mu \rightarrow \bar{\nu}_{\mu}+\nu_{e}+e$ decay chain. The observed ratio of $\mathrm{GeV}$ neutrinos is closer to one as a consequence of oscillations. Above several $\mathrm{GeV}$, however, the abundance of $\nu_{e}$ decreases rapidly as the higher energy muons reach the ground before decaying. In the $\mathrm{TeV}$ range and above, the intensity of atmospheric $\nu_{e}$ is only $3-4 \%$ of $\nu_{\mu}$, the primary channel for their production being one of the semileptonic decays of $\mathrm{K}_{\mathrm{L}}$. Because of their low rates and the requirement for containment, it has been difficult to identify cascade like events in the multi-TeV range with the partially completed IceCube. Preliminary results on high-energy cascades in IceCube will be discussed below.

In the last two construction seasons a subarray of more closely spaced sensors with higher quantum efficiency was deployed in the deep central region of IceCube (IceCube, 2012a). With this subarray, called DeepCore, it is now possible to measure the flux of electron neutrinos in the 0.1-1 TeV energy range using the increased sensitivity of the denser subarray to low-energy events. Preliminary results (Ha, 2012) are shown in the left panel of Fig. 3. Quantitative evaluation of the intensity of $\nu_{e}$ will depend on accumulating sufficient statistics to subtract the neutral current contributions and the background of low-energy, charged-current $\nu_{\mu}$ events remaining in the sample. The ratios of the different contributions are obtained from simulation starting from two different calculations of the flux of atmospheric neutrinos, by Barr et al. (2004) and by Honda et al. (2007).

The DeepCore array also provides better sensitivity for short muon tracks, leading to the possibility of reconstructing the directions of charged current $\nu_{\mu}$ events with energies between 10 and $100 \mathrm{GeV}$. The right panel of Fig. 3 compares the angular dependence of these events to what is expected without oscillations (red) and with oscillations assuming the standard oscillation parameters (black). The suppression of $\nu_{\mu}$ with long pathlengths through the Earth $(\cos (\theta)$ near -1$)$ is clearly seen (Gross, 2012). 

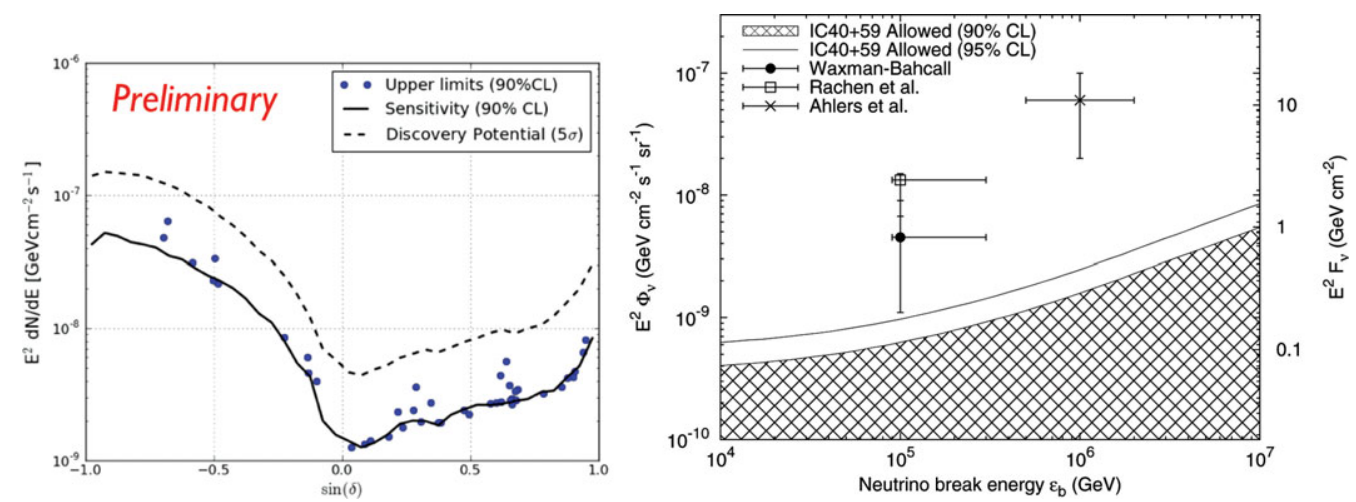

Figure 4. Left: Limits on neutrinos from selected point sources (vs sine of declination). Right: Upper limits on neutrinos from GRBs.

\subsection{Search for point sources of astrophysical neutrinos}

The most direct approach to searching for sources of neutrinos with IceCube is to map the sky in neutrino-induced muons (IceCube, 2011c). A sky map of the significance relative to background is created for the whole sky. For the Northern sky (directions from below the horizon at the South Pole) the background after event selection is produced by atmospheric $\nu_{\mu}$ with contamination from mis-reconstructed events at the per cent level. The Southern sky is dominated by the high rate of atmospheric muons from above, so the selection criteria are quite different and include the veto probability from IceTop. In both cases an unbinned maximum likelihood approach is used that accounts for the features of the backgrounds, which are different for the Northern and Southern skies. The likelihood procedure takes into account known features of the background, including the steep energy spectrum and the angular distribution of the atmospheric neutrinos and muons.

Results are also given for a selected list that includes 13 galactic sources such as supernova remnants and microquasars and 30 extragalactic sources, mostly AGNs. The left panel of Fig. 4 shows the limits for the selected sources obtained with two years of the partially completed IceCube running with 40 strings in 2008-09 and with 59 strings in 2009-2010 (Aguilar, 2012). Although no highly significant sources have been identified yet, it is interesting to note that the limits summed over the Northern sky are approaching the level of $10^{-9} \mathrm{GeV} \mathrm{cm}^{-2} \mathrm{sr}^{-1} \mathrm{~s}^{-1}$ at which neutrinos are expected if they are produced at the level suggested by Eq. 2.4.

There are also dedicated searches for flaring sources (IceCube, 2012b), both looking for correlations with flaring activity observed in gamma-rays and by looking for sequences of neutrinos correlated in time. A system has been set up for sending alerts for rapid followup by optical telescopes when two or more neutrinos from nearly the same direction occur within a short time interval (IceCube, 2012c).

\subsection{Neutrinos from gamma-ray bursts}

Gamma ray bursts provide sharp time stamps and in most cases a direction in which to look for accompanying neutrinos. IceCube looked for high energy neutrinos in association with 300 bursts that occurred during the same two year period mentioned above (IC-40 and IC59). No neutrinos were seen (IceCube, 2012d). The right panel of Fig. 4 shows the flux upper limits as a function of the break energy in the neutrino spectrum, which depends on the assumed bulk Lorentz factor of the GRB jet and the observed 
gamma-ray spectrum of the burst. The limit is significantly lower than predictions (Rachen \& Meszaros, 1998, Ahlers et al., 2011) normalized by the assumption that GRBs are the source of the extragalactic cosmic rays, including the original calculation of Waxman \& Bahcall (1997). The result also rules out a flux at the level predicted by the model of Guetta et al. 2004. However, the parameters of this model have since been questioned by Hummer, Baerwald \& Winter (2012) who suggest the neutrino flux prediction could be an order of magnitude lower.

The right ordinate of the GRB limit in Fig. 4 shows upper limits on the fluence accumulated during the 300 time windows around each burst. The average duration in the sample was 28 seconds per burst. Assuming 667 GRBs per year in the whole sky, a limit on the diffuse flux from all gamma-ray bursts is calculated and shown on the left ordinate. This more general limit depends on the shape of the model but not its normalization. It is interesting that it is at the expected level of Eq. 2.4.
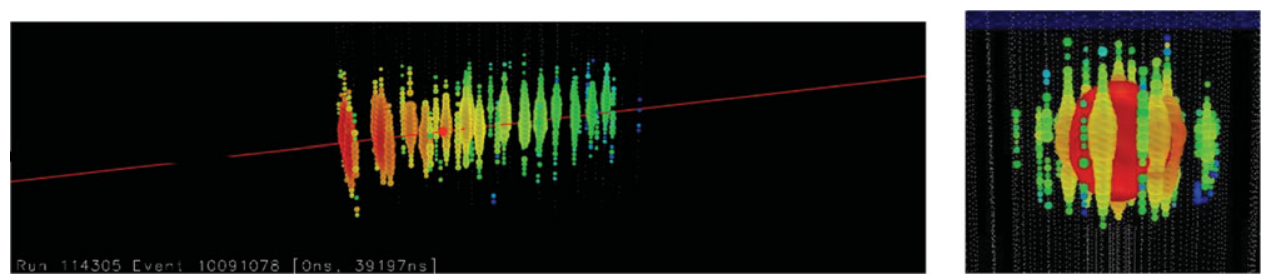

Figure 5. Left: Large track event; Right: Large cascade event.

\subsection{Neutrinos from unresolved sources}

Because neutrinos are not absorbed during propagation, the flux at Earth will receive equal contributions from each spherical shell out to the Hubble radius. It is therefore important to look for neutrinos from all directions (Lipari, 2008). This "diffuse" analysis is the same that produces the atmospheric neutrino spectrum as a byproduct (Fig. 2, right). The normalization and slope of the atmospheric spectrum and the fraction of prompt neutrinos are fitted nuisance parameters of the analysis. The upper horizontal, solid line in Fig. 2 (right) is the upper limit for IceCube in 2009-10 (IC-59) (Schukraft, 2012). The lower line is the Waxman-Bahcall limit on the flux of astrophysical neutrinos (Waxman, 2011), which is essentially at the level of the observed cosmic-ray energy content of Eq. 2.1. The bound is an upper limit derived by Waxman \& Bahcall (1998) from the observation that for a transparent (efficient) source there should not be more energy in neutrinos than in the cosmic-rays that produced them. The IceCube upper limit is a factor of two higher than the sensitivity of the analysis. One of the largest events from this analysis is shown in the left panel of Fig. 5.

\subsection{Cosmogenic neutrinos}

If the energy spectrum of extragalactic cosmic rays extends above the threshold for photopion production on the microwave background, neutrinos will be produced as the cosmic rays propagate over cosmic distances (Berezinsky \& Zatsepin, 1969). The paper of Kotera, Allard \& Olinto (2011) is an overview of the possible levels of neutrino production, which depend on factors such as the cosmological evolution of the power of the sources and the spectrum and composition of the accelerated particles. Expectations for event rates in the completed IceCube are at the level of one event per year (IceCube, 2011d).

A search with two years of data (June 2010 - May 2012, IC-79 and IC-86) (Ishihara, 2012) found no events in the energy range expected for cosmogenic neutrinos (10 to $1000 \mathrm{PeV}$ ). However, two cascade-like events were found above one PeV, slightly above 
the threshold in total observed photo-electrons for the analysis. Preliminary energies estimated for the cascades are 1.1 and $1.3 \mathrm{PeV}$ with a total uncertainty (statistical plus systematic) of $35 \%$. The $1.1 \mathrm{PeV}$ event is shown in the right panel of Fig. 5. A study is under way in preparation for unblinding the entire high energy data sample to investigate the spectrum below the threshold for the cosmogenic analysis.
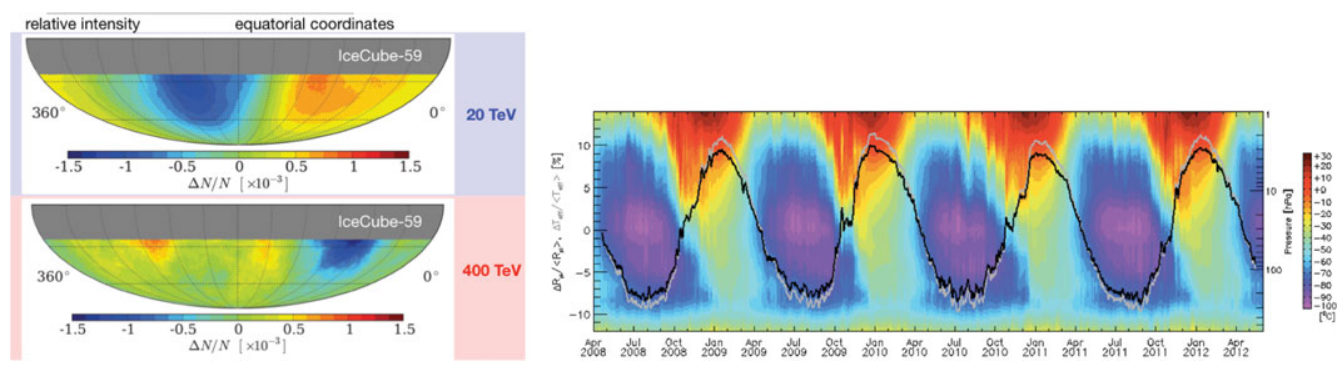

Figure 6. Left: Anisotropy in the Southern hemisphere; Right: Seasonal variations of IceCube rates over four years.

\section{Broader aspects of IceCube and Outlook}

As a large particle detector, IceCube has a broad scientific scope ranging from studies of cosmic-ray spectrum (IceCube, 2012e) and composition (IceCube, 2012f) to searches for dark matter (Icecube, 2012g) and for new physics such as magnetic monopoles (IceCube, 2012h) and violation of Lorentz invariance (IceCube, 2010a). Counting rates in IceTop tanks provide novel spectral information about particles from solar flares that produce ground level events (IceCube, 2008). Counting rates of DOMs in the stable, quiet, cold environment of the deep ice continuously monitor for $\sim 10 \mathrm{MeV}$ neutrinos from supernovas in our galaxy (IceCube, 2011e)

As the largest deep detector, IceCube detects $\mathrm{TeV}$ atmospheric muons at an unprecedented rate. With the completed IceCube, the rate of events that trigger 8 or more DOMs is $2.2 \mathrm{kHz}$ with a seasonal variation of $\pm 9 \%$ after a $10 \%$ correction for accidental coincidences. Directions and energies are reconstructed online to sufficient accuracy to map cosmic-ray anisotropies that reflect scales of the outer heliosphere and nearby interstellar medium (IceCube, 2012i). The same muons can be used to probe seasonal and short-term variations in the stratosphere with unprecedented precision as the atmosphere expands and contracts in response to changes in temperature (IceCube, 2011f). Muon maps and seasonal variations are illustrated in Fig. 6.

Data taking with the completed IceCube detector began in May 2011, and analysis of data with the full detector is just getting fully underway. Indications of high-energy astrophysical neutrinos are already beginning to appear. The detector is stable with negligible loss of hardware in the ice so far, so it should be able to operate for the extended period of a decade or more needed to accumulate a good sample of neutrinos.

Acknowledgment This work was supported in part by NSF-ANT-0856253.

\section{References}

Astrophysics in Antarctica 1989, AIP Conf. Proc. 198 (ed. Dermott J. Mullan, Martin A. Pomerantz \& Todor Stanev).

Ageron, M. et al. 2011, arXiv:1104.1607v2.

Aguilar, J.A. 2012 (for the IceCube Collaboration), Proc. 9th Workshop on Science with the New Generation of High Energy Gamma-ray Experiments (SciNeGHE 2012).

Ahlers, M., et al. 2005, Phys. Rev. D72, 023001.

Ahlers, M., M. Gonzalez-Garcia \& F. Halzen 2011, Astropart. Phys. 35, 87-94. 
Allard, D. et al. 2011, JCAP 10-033.

Babson, J. et al. 1990, Phys. Rev. D42, 3613-3620.

Balkanov, V. A. et al. 1999, Astropart. Phys. 12, 75-86.

Barr, G. D. et al. 2006, Phys. Rev. D74, 094009.

Berezinsky, V.S. \& G.T. Zatsepin, Phys. Lett. 28 B, 423.

Berezinsky, V., A. Gazizov \& S. Grigorieva 2006, Phys. Rev. D74, 043005.

Bionta, R. M. et al. 1987, Phys. Rev. Lett. 58, 1494-1496.

Fukuda, Y. et al. 1998, Phys. Rev. Lett. 81, 1562-1567.

Gaisser, T. K. 2012, Astropart. Phys. 35, 801-806.

Greisen, K. 1960, Ann. Rev. Nuclear Sci. 10, 63-108.

Gross, A. 2012 (for the IceCube Collaboration), Neutrino 2012.

Guetta, D. et al. 2004, Astropart. Phys. 20, 429.

Ha, C. 2012 (for the IceCube Collaboration), arXiv:1209.0698v1.

Halzen, F., J. Learned \& T. Stanev 1989, in AIP Conf. Proc. 198, 39-51.

Halzen, F. 2012, these Proceedings.

Hirata, K. et al., 1987, Phys. Rev. Lett. 58, 1490-1493.

Honda, M. et al. 2007, Phys. Rev. D75, 043006.

Hummer, S., P. Baerwald \& W. Winter 2012, Phys. Rev. Letters 108, 231101.

IceCube Collaboration 2008 (R. Abbasi et al.), Ap.J. 689, L65-68.

IceCube Collaboration 2009a (R. Abbasi et al.), Phys. Rev. D79, 062001.

IceCube Collaboration 2009b (R. Abbasi et al.), Phys. Rev. D79, 102005.

IceCube Collaboration 2010a (R. Abbasi et al.), Phys. Rev. D82, 112003.

IceCube Collaboration 2010b ( R. Abbasi et al.), Astropart. Phys. 34, 48-58.

IceCube Collaboration 2011a (R. Abbasi et al.), Phys. Rev. D83, 012001.

IceCube Collaboration 2011b (R.Abbasi et al.), Phys. Rev. D84, 082001.

IceCube Collaboration 2011c (R. Abbasi et al.), Ap.J. 732, 18.

IceCube Collaboration 2011d (R. Abbasi et al.), Phys. Rev. D83, 092003.

IceCube Collaboration 2011e (R. Abbasi et al.), Astronomy 83 Astrophysics 535, A109.

IceCube Collaboration 2011f (R. Abbasi et al.), arXiv:1111.2735 (Proc. 32nd ICRC, Beijing).

IceCube Collaboration 2012a (R. Abbasi et al.), Astropart. Phys. 35, 615-624.

IceCube Collaboration 2012b (R. Abbasi et al.), Ap.J. 744, 1.

IceCube Collaboration 2012c (R. Abbasi et al.), Astron. ES Astrophys. 539, A60.

IceCube Collaboration 2012d (R. Abbasi et al.), Nature 484, 351-354.

IceCube Collaboration 2012e (R. Abbasi et al.), arXiv:1202.3039 (submitted to Astropart. Phys.)

IceCube Collaboration 2012f (R. Abbasi et al.), arXiv:1207.3455 (submitted to Astropart. Phys.).

IceCube Collaboration 2012g (R. Abbasi et al.), Phys. Rev D85, 042004.

IceCube Collaboration 2012h (R. Abbasi et al.), arXiv:1208.4861 (submitted to Phys. Rev. D). IceCube Collaboration 2012i (R. Abbasi et al.), Ap.J. 745, 45.

Ishihara, A. 2012 (for the IceCube Collaboration) to appear in Proc. Neutrino 2012.

Jelley, N., A. B. McDonald \& R. G. H. Robertson 2009, Ann. Revs. Nucl. Part. Sci. 59, 431-465.

Karle, A. 2012, these Proceedings.

Kotera, K., D. Allard \& A. V. Olinto 2011, JCAP 10-013.

Learned, J. G. \& S. Pakvasa 1995, Astropart. Phys. 3, 267-274.

Markov, M. A. 1960, Proc. Annual International Conference on High Energy Physics at Rochester.

Lipari, P. 2008, Phys. Rev. D 78, 083011.

Rachen, J.P., \& P. Meszaros 1998 in Fourth Huntsville Gamma-Ray Burst Symposium (A.I.P. Conf. Proceedings 428, eds. C.A. Meegan, R.D. Preece \& T.M. Koshut) 776-780.

Reines, F. 1960, Ann. Rev. Nuclear Sci. 10, 1-26.

Schukraft, A. 2012 (for the IceCube Collaboration), presented at NOW2012.

Waxman, E. \& J. Bahcall 1997, Phys. Rev. Letters 78, 2292.

Waxman, E. \& J. Bahcall 1998, Phys. Rev. D59 012002.

Waxman, E. 2011 arXiv:1101.1155v1. 